BMJ Open

Diabetes

Research

\& Care

\section{Cardiovascular outcomes of type 2 diabetic patients treated with SGLT-2 inhibitors versus GLP-1 receptor agonists in real-life}

\author{
Enrico Longato, ${ }^{1}$ Barbara Di Camillo (i) , ${ }^{1}$ Giovanni Sparacino (1) , \\ Lorenzo Gubian, ${ }^{2}$ Angelo Avogaro, ${ }^{3}$ Gian Paolo Fadini (D) ${ }^{3}$
}

To cite: Longato $\mathrm{E}$, Di Camillo B, Sparacino G, et al. Cardiovascular outcomes of type 2 diabetic patients treated with SGLT2 inhibitors versus GLP-1 receptor agonists in real-life. BMJ Open Diab Res Care 2020;8:e001451. doi:10.1136/ bmjdrc-2020-001451

\section{- Additional material is published online only. To view please visit the journal online (http://dx.doi.org/10.1136/ bmjdrc-2020-001451).}

Received 8 April 2020 Revised 12 May 2020 Accepted 24 May 2020

Check for updates

(C) Author(s) (or their employer(s)) 2020. Re-use permitted under CC BY-NC. No commercial re-use. See rights and permissions. Published by BMJ.

${ }^{1}$ Department of Information Engineering, Università degli Studi di Padova, Padova, Veneto, Italy

${ }^{2}$ Azienda Zero, Regione Veneto, Padova, Veneto, Italy ${ }^{3}$ Department of Medicine, Università degli Studi di Padova, Padova, Veneto, Italy

Correspondence to Professor Gian Paolo Fadini; gianpaolofadini@hotmail.com

\section{ABSTRACT}

Introduction Sodium glucose cotransporter-2 inhibitors (SGLT2i) and glucagon-like peptide-1 receptor agonists (GLP-1RA) protect type 2 diabetic (T2D) patients from cardiovascular events, but no trial has directly compared their cardiovascular effects. We aimed to address this gap using real-world data.

Research design and methods We performed a retrospective real-world study on a population of $\sim 5$ million inhabitants from North-East Italy. We identified T2D patients who received new prescription of SGLT2i or GLP-1RA from 2014 to 2018. SGLT2i and GLP-1RA initiators were matched $1: 1$ by propensity scores. The primary outcome was a composite of all-cause death, myocardial infarction, and stroke (three-point major adverse cardiovascular events (3P-MACE)). Secondary endpoints were each component of the primary endpoint, hospitalization for heart failure (HF), revascularization, hospitalization for cardiovascular causes, and adverse events.

Results From a population of 330193 diabetic patients, we followed 8596 SGLT2i and GLP-1RA matched initiators for a median of 13 months. Patients in both groups were on average 63 years old, $63 \%$ men, and $18 \%$ had preexisting cardiovascular disease. T2D patients treated with SGLT2i versus GLP-1RA, experienced a lower rate of 3P-MACE (HR 0.68; 95\% Cl 0.61 to $0.99 ; \mathrm{p}=0.043$ ), myocardial infarction (HR 0.72; $95 \% \mathrm{Cl} 0.53$ to 0.98 ; $\mathrm{p}=0.035$ ), hospitalization for HF (HR 0.59; $95 \% \mathrm{Cl} 0.35$ to $0.99 ; p=0.048$ ), and hospitalization for cardiovascular causes (HR 0.82; 95\% Cl 0.69 to 0.99; $p=0.037$ ). Adverse events were not significantly different between the two groups.

Conclusions In the absence of dedicated trials, this observational study suggests that SGLT2i may be more effective than GLP-1RA in improving cardiovascular outcomes of T2D.

Trial registration number NCT04184947.

\section{INTRODUCTION}

Despite a global decline in the burden of cardiovascular disease (CVD), patients with type 2 diabetes (T2D) still experience high rates of major adverse cardiovascular events (MACE). ${ }^{1}$ Tackling the diabetes-associated

\section{Significance of this study}

What is already known about this subject?

- Glucagon-like peptide-1 receptor agonists (GLP1RA) and sodium glucose cotransporter-2 inhibitors (SGLT2i) both protect against adverse cardiovascular outcomes of type 2 diabetes (T2D).

- A direct comparison of cardiovascular outcomes in patients treated with GLP-1RA versus SGLT2i is missing.

\section{What are the new findings?}

- We compared cardiovascular outcomes of patients with T2D who initiated a GLP-1RA or SGLT2i in routine clinical practice.

- We found that rates of major adverse cardiovascular events and hospitalization for heart failure were lower in patients with T2D initiating an SGLT2i versus a GLP-1RA.

- Results were highly consistent in the intention to treat and as treated datasets, as well as in sensitivity analyses.

How might these results change the focus of research or clinical practice?

- In the absence of direct comparative trials, this observational study suggests that use of SGLT2i might be associated with a lower cardiovascular risk than use of GLP-1RA.

- Confirmation of these results are needed before they can be incorporated into clinical decision-making.

cardiovascular risk thus remains a major challenge.

In cardiovascular outcome trials (CVOTs), sodium glucose cotransporter-2 inhibitors

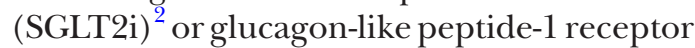
agonists (GLP-1RA) ${ }^{3}$ reduced MACE rates in T2D patients on a background of standard medical therapy. Though most T2D patients included in earlier trials had established CVD, the Dapagliflozin Effect on Cardiovascular Events (DECLARE) ${ }^{4}$ and Researching Cardiovascular Events with a Weekly Incretin 
in Diabetes (REWIND) studies $^{5}$ recently showed that cardiovascular benefits of SGLT2i and GLP-1RA may extend to T2D patients without CVD. Some differences between these two classes of drugs have emerged with regards to the type of cardiovascular events they prevent. ${ }^{6}$ While GLP-1RA mostly tackle atherosclerotic CVD, SGLT2i exert much of their protective effects against heart failure (HF). Both classes have demonstrated potential to delay mortality (especially cardiovascular), ${ }^{23}$ and to reduce the risk of renal disease. ${ }^{37}$ Remarkably, cardiovascular and renal benefits of SGLT2i and GLP1RA have been confirmed in large observational realworld studies. ${ }^{8-12}$

Despite these similarities, SGLT2i and GLP-1RA differ in mechanism of action, administration regimen, and safety/tolerability. SGLT2i are administered orally and induce urinary loss of sodium and glucose.$^{13}$ Their most common side effects are genito-urinary tract infections (GUTI), sometimes leading to transient discontinuation. Rarer but serious adverse events include dehydration/ hypovolemia, acute kidney injury (AKI), diabetic ketoacidosis, bone fractures, amputations, and Fournier's gangrene. ${ }^{14}$ GLP-1RA are administered subcutaneously and potentiate meal-induced insulin release. Their most common side effects are gastrointestinal, sometimes driving discontinuation or intolerance, while pancreatitis and pancreatic cancer are potentially serious adverse events. ${ }^{15}$ These differences can result in the preference of SGLT2i or GLP-1RA according to patient characteristics.

A prior study on Italian diabetic outpatients showed that the probability of achieving a simultaneous improvement in glycated hemoglobin (HbA1c), blood pressure and body weight was equal with the two treatments. ${ }^{16}$ Yet, no clinical trial has addressed the question of which of the two classes of drugs is more effective in protecting T2D patients from adverse cardiovascular outcomes, nor any such trial is planned. In the absence of evidence from clinical trials, data from observational studies help in generating hypotheses and designing dedicated CVOTs. ${ }^{17}$ Although they cannot substitute for CVOTs, ${ }^{18}$ observational studies can provide medium-level evidence to guide clinical decision making.

In this observational retrospective study, we compared cardiovascular outcomes and adverse events of patients who had been newly treated with SGLT2i or GLP-1RA from 2014 to 2018.

\section{RESEARCH DESIGN AND METHODS}

\section{Data source and cohort identification}

Veneto is a populous region in North-East Italy, with around 5 million inhabitants and an almost entirely public healthcare system. In addition to an extensive administrative claims repository, needed for reimbursement and governance purposes, the Veneto region has been maintaining a regional Health Information Exchange (rHIE) system to share all documents produced within its healthcare facilities. ${ }^{19}$
The study protocol has been posted online on ClinicalTrials.gov. In this region-wide, retrospective, observational study, we referenced Veneto's register of healthcare beneficiaries ${ }^{20}$ to identify Italian citizens, residing in the region, who had been registered as beneficiaries for at least 1 year between 1 January 2011 and 30 September 2018, or time of death. The available data comprised biographical information, exemptions from copayment, and all administrative claims generated by all subjects in relation to prescription, medicine dispensations, procedures performed during hospital stay, and diagnostic codes at hospital discharge. Due to the lack of a central diabetes registry, we used an algorithm for the identification of diabetes via administrative claims ${ }^{21}$ : validated against clinical diagnosis, the algorithm had $95.7 \%$ sensitivity, $87.9 \%$ specificity, and $97.6 \%$ precision. We then identified those who started a new therapy regimen with SGLT2i (dapagliflozin, empagliflozin or canagliflozin) or GLP-1RA (exenatide, liraglutide, lixisenatide, dulaglutide) in the observation period. As these drugs were not reimbursed for type 1 diabetes, we can assume that all patients included in the analysis had T2D. We defined the index date as the start of a new therapy regimen with either SGLT2i (Anatomical Therapeutic Classification (ATC) codes A10BK, A10BD15, A10BD16, A10BD19, A10BD20, or A10BD21) or GLP-1RA (ATC A10BJ). We allowed for a 7-month delay between the first visible A10 prescription in the database and therapy initiation to minimize the risk of including therapies that were, in fact, ongoing when the subject first appeared in the claims database. We only included subjects whose index date was in or after January 2014 when SGLT2i formally received first marketing authorization approval. Our primary analysis conformed to an 'as treated' (AT) approach, where we followed all subjects until therapy discontinuation (defined as 7 months after the last refill; see below), outcome occurrence, or exit from the database (last observation); we also performed a sensitivity analysis using an 'intention to treat' (ITT) approach, that is, disregarding therapy discontinuation.

We verified the adequacy of a 7 -month window by comparing therapy discontinuation as reported in the rHIE for a minority of subjects versus their refill patterns. We found that $>90 \%$ of the subjects who stopped refilling their SGLT2i and GLP-1RA prescriptions for 7 months had, in fact, been instructed to discontinue their therapy regimen by the diabetologist. Thus, not observing a prescription of SGLT2i or GLP-1RA between the first A10-class medication and the index date for 7 months almost certainly means that therapy had not started yet; similarly, not observing a refill for 7 months indicates that therapy had been discontinued.

To avoid reverse causality-which can occur when drugs with expected cardioprotective effects are prescribed to patients because they are perceived to be at very high risk of imminent cardiovascular events, or in case of planned coronary, carotid interventions, which can generate 
Table 1 Baseline claims-based characteristics after matching

\begin{tabular}{|c|c|c|c|c|}
\hline & SGLT2i (N=4298) & GLP-1RA (N=4298) & SMD* & $P$ value \\
\hline \multicolumn{5}{|l|}{ Demographics } \\
\hline Age at index date (years) & $62.7(9.2)$ & $62.8(9.7)$ & -0.01 & 0.230 \\
\hline Female sex $(\%)$ & 37 & 36.9 & $<0.01$ & 0.964 \\
\hline Claims-based history length $\ddagger$ (months) & $60.4(11.9)$ & $60.3(14.5)$ & $<0.01$ & 0.004 \\
\hline Claims-based diabetes duration§ (months) & $113(65.3)$ & $113(66.2)$ & $<0.01$ & 0.491 \\
\hline \multicolumn{5}{|l|}{ Risk factors } \\
\hline Hypertension (\%) & 81.7 & 83 & -0.03 & 0.134 \\
\hline Dyslipidemia (\%) & 75.2 & 74 & 0.03 & 0.215 \\
\hline \multicolumn{5}{|l|}{ Macrovascular complications } \\
\hline Peripheral circulatory complications (\%) & 2.3 & 2 & 0.02 & 0.502 \\
\hline Infarction (\%) & 8.1 & 7.6 & 0.02 & 0.470 \\
\hline Ischemic heart disease (\%) & 13.1 & 12.7 & 0.01 & 0.629 \\
\hline Stroke or TIA (\%) & 4.5 & 4.1 & 0.02 & 0.456 \\
\hline Heart failure (\%) & 2.8 & 2.8 & $<0.01$ & 1.000 \\
\hline Cardiovascular disease (\%) & 18.3 & 17.7 & 0.02 & 0.465 \\
\hline \multicolumn{5}{|l|}{ Microvascular complications } \\
\hline Neurological complications (\%) & 0.6 & 0.4 & 0.02 & 0.439 \\
\hline Ocular complications (\%) & 0.5 & 0.5 & $<0.01$ & 1.000 \\
\hline Renal complications (\%) & 0.4 & 0.5 & -0.01 & 0.748 \\
\hline Chronic kidney disease (\%) & 1.4 & 1.8 & -0.03 & 0.198 \\
\hline Severe hypoglycemia (\%) & 1.1 & 0.9 & 0.03 & 0.281 \\
\hline \multicolumn{5}{|l|}{ Comorbidities } \\
\hline Chronic pulmonary disease (\%) & 33.2 & 33.8 & -0.01 & 0.552 \\
\hline Systemic inflammatory disease (\%) & 2.2 & 2.2 & $<0.01$ & 1.000 \\
\hline Cancer (\%) & 10.6 & 10.7 & $<0.01$ & 1.000 \\
\hline Charlson comorbidity index & $0.5(1.2)$ & $0.5(1.1)$ & -0.01 & 0.107 \\
\hline \multicolumn{5}{|l|}{ Glucose lowering medications } \\
\hline Different A10B therapies $(n) \uparrow$ & $2.3(1.3)$ & $2.3(1.2)$ & $<0.01$ & 0.392 \\
\hline Ever used insulin (\%) & 33.9 & 33.6 & $<0.01$ & 0.837 \\
\hline Any insulin (\%) & 28.5 & 29.1 & -0.01 & 0.552 \\
\hline Long-acting insulin (\%) & 27.9 & 28.1 & $<0.01$ & 0.904 \\
\hline Fast-acting insulin (\%) & 12.8 & 13.4 & -0.02 & 0.388 \\
\hline Metformin (\%) & 89.0 & 89.0 & $<0.01$ & 0.973 \\
\hline Sulfonylureas (\%) & 43.6 & 44.0 & -0.01 & 0.794 \\
\hline DPP4i (\%) & 30.8 & 31.3 & -0.01 & 0.624 \\
\hline Pioglitazone (\%) & 12.7 & 12.4 & 0.01 & 0.721 \\
\hline \multicolumn{5}{|l|}{ Other therapies } \\
\hline ACE inhibitors (\%) & 70.2 & 71.3 & -0.03 & 0.255 \\
\hline Diuretics (\%) & 18.2 & 18.7 & -0.01 & 0.578 \\
\hline Beta blockers (\%) & 35.6 & 36.2 & -0.01 & 0.574 \\
\hline Other antihypertensives (\%) & 7.5 & 7.7 & -0.01 & 0.684 \\
\hline Statins (\%) & 64.5 & 64.5 & $<0.01$ & 1.000 \\
\hline Fibrates or omega-3 (\%) & 12.6 & 12.2 & 0.01 & 0.578 \\
\hline PCSK9 inhibitors (\%) & 0 & 0 & 0 & $\mathrm{~N} / \mathrm{A}$ \\
\hline Ezetimibe (\%) & 3.9 & 3.9 & $<0.01$ & 1.000 \\
\hline
\end{tabular}


Table 1 Continued

\begin{tabular}{lcccc}
\hline & SGLT2i (N=4298) & GLP-1RA (N=4298) & SMD $^{*}$ & P value† \\
\hline Platelet aggregation inhibitors (\%) & 35.0 & 35.9 & -0.02 & 0.417 \\
\hline Clinical-laboratory data & & & & \\
\hline Fasting glucose $(\mathrm{mg} / \mathrm{dL})$ & $168.5(48.4)$ & $161.5(42.3)$ & 0.15 & 0.013 \\
\hline HbA1c $(\%)$ & $8.0(1.1)$ & $7.8(0.9)$ & 0.21 & $<0.001$ \\
\hline Total cholesterol $(\mathrm{mg} / \mathrm{dL})$ & $168.6(37.8)$ & $168.8(37.2)$ & -0.01 & 0.497 \\
\hline HDL cholesterol $(\mathrm{mg} / \mathrm{dL})$ & $47.3(13.9)$ & $47.9(12.8)$ & -0.04 & 0.142 \\
\hline LDL cholesterol $(\mathrm{mg} / \mathrm{dL})$ & $93.6(33.6)$ & $93.0(32.4)$ & 0.02 & 0.326 \\
\hline Triglycerides $(\mathrm{mg} / \mathrm{dL})$ & $137.1(56.6)$ & $139.4(57.4)$ & -0.04 & 0.237 \\
\hline Systolic blood pressure $(\mathrm{mm} \mathrm{Hg})$ & $141.9(20.9)$ & $141.9(18.7)$ & $<0.01$ & 0.371 \\
\hline Diastolic blood pressure $(\mathrm{mm} \mathrm{Hg})$ & $81.2(10.6)$ & $81.1(10.7)$ & $<0.01$ & 0.450 \\
\hline
\end{tabular}

Unless otherwise indicated, medication-related variables were calculated starting from 12 months before the index date. Pre-existing conditions were assessed with all available data up to the index date. SMD values reported as ' $<0.01$ ' are, in fact, $<0.01$ and $>-0.01$. *SMDs (positive if SGLT2i greater).

$+\chi^{2}$ test for categorical variables (expressed as \%), Mann-Whitney test otherwise.

$\ddagger$ Time interval between the first available claim and the index date.

§Time interval between the first claim or exemption from copayment indicating diabetes and the index date.

ПComputed using all available data up to the index date.

ACE, angiotensin converting enzyme; DPP4i, Dipeptidyl peptidase-4 inhibitors; GLP-1RA, glucagon-like peptide-1 receptor agonists; HbA1c, glycated hemoglobin; HDL, high density lipoprotein; LDL, low density lipoprotein;; PCSK9, proprotein convertase subtilisin kinase-9; SGLT2i, sodium glucose cotransporter-2 inhibitors; SMD, standardized mean difference; TIA, transient ischemic attack.

peri-procedural events-we excluded all events occurring within the first and second months after the index date.

\section{Outcome definition}

The primary endpoint of this study was the three-point MACE (3P-MACE) composite outcome, defined as the first occurrence of myocardial infarction, stroke, or death. All-cause death was considered because causes of death were not available. Secondary endpoints were each of the 3P-MACE components, hospitalization for HF, arterial revascularizations, and hospitalization for cardiovascular causes. We identified and dated all cardiovascular events based on the first six diagnosis codes (one primary, five secondary) and admission time reported in the hospital discharge claims database, that is, by mapping ICD-9-CM (international classification of diseases ninth revision clinical modification) codes to the appropriate outcomes as follows: diagnosis codes 410-414 to infarction, 431-436 to stroke, 428 to hospitalization for HF, and 390-459 to hospitalization for cardiovascular causes; procedure codes 00.55, 00.61-00.66, 36.03, 36.06-36.07, 36.1, 38.48, $39.50,39.52,39.71$, and 39.90 to revascularization. We expressed all outcome times in "months since the index date' to compensate for the hiding of the precise date of death during the anonymization process.

\section{Propensity score matching and statistical analysis}

To address the imbalance between the two groups, we performed 1:1 propensity score matching (PSM) by applying the nearest neighbor method to the logit distance with a maximum calliper of $0.0023 \mathrm{SD}$ and no replacement. We included 39 variables, belonging to five general categories, in the logistic regression model used to estimate propensity scores (PS): subject information (four variables), that is, age at index date, sex, time (in months) between the first observable claim and the index date (hereafter called 'claims-based history length'), and time between the first claim or exemption from copayment indicative of known diabetes and the index date (hereafter called "claims-based diabetes duration'); antidiabetic agents use (two variables): insulin use in the patient's history, number of different A10B-class drugs (ie, 'blood glucose lowering drugs, excluding insulins') in the entire patient's history; antidiabetic agents, as observed in the year preceding the index date (six variables); other medication (nine variables); pre-existing conditions (18 variables, including Charlson's comorbidity index computed as described) ${ }^{22}{ }^{23}$ The full list of variables is given in table 1 and their definitions using claims in online supplementary table S1.

To evaluate the effectiveness of PSM, we performed univariate tests on each variable $\left(\chi^{2}\right.$ for binary variables; Mann-Whitney's U tests for all others). If no significant differences were apparent (ie, if the $\mathrm{p}$ value was greater than the conventional significance level of 0.05 ) or if their size was negligible (ie, if the corresponding absolute standardized mean difference was $<0.10$ ), we considered that variable successfully matched. Then, we tested whether a satisfactory degree of matching would also be found in laboratory test results, using a strategy already employed before. ${ }^{8}$ To this end, we identified subgroups of subjects whose laboratory test results concerning fasting glucose, HbA1c, total cholesterol, high density lipoprotein (HDL) cholesterol, and triglycerides were available in the rHIE 


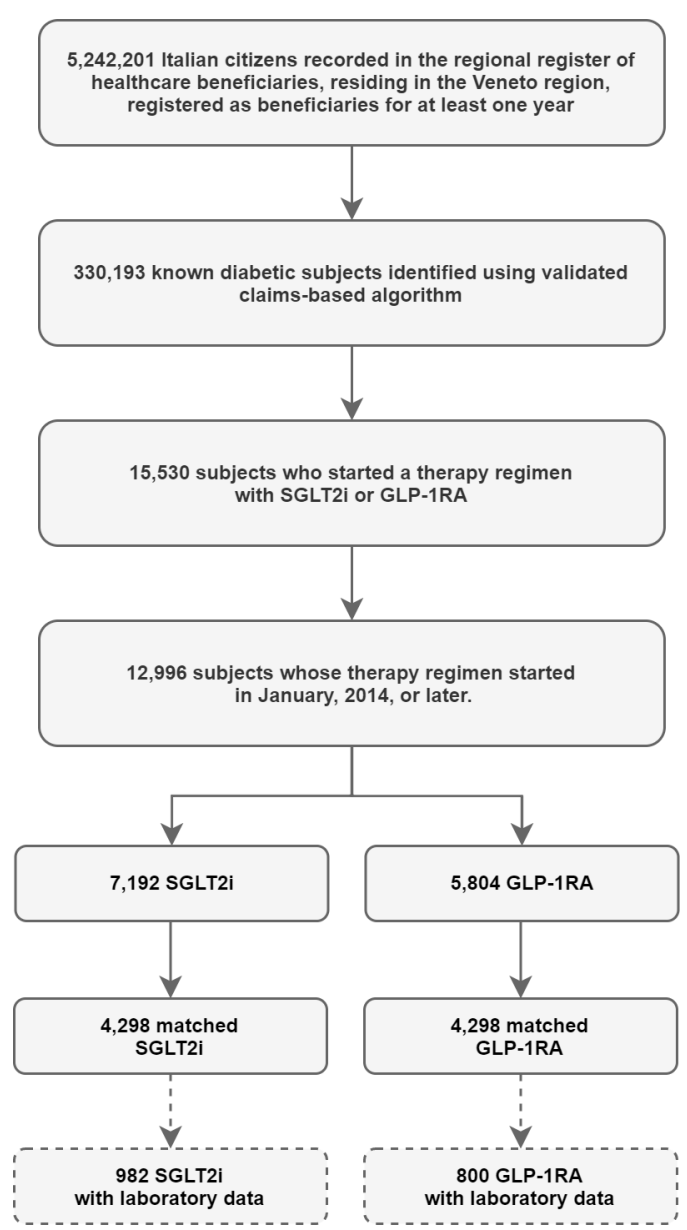

Figure 1 Study flowchart. Visual description of the general framework of the study with sample size after each filtering step. GLP-1RA, glucagon-like peptide-1 receptor agonists; SGLT2i, sodium glucose cotransporter-2 inhibitors.

near the index date. Among all available results, we isolated the ones closest to the index date. Updated values of laboratory variables were also collected, when available, from the index date to censoring, as close as possible to the outcome or censoring date.

The primary analysis was a Cox regression on the matched populations to evaluate the HRs between the SGLT2i and GLP-1RA groups with respect to the occurrence of the primary and secondary cardiovascular endpoints described above, following the AT approach. An ITT analysis was performed separately.

We also performed supplementary, explorative analyses, both using Cox regression. In the first, we compared HRs for all outcomes after stratifying according to preexisting CVD. In the second, we considered only the primary endpoint (3P-MACE) and estimated the unadjusted HRs after the following stratifications: female versus male subjects, older versus younger subjects, subject treated versus not treated with insulin, statins, and diuretics. To complement both supplementary analyses, we also fit interaction models to assess the $p$ value associated with the interaction terms (ie, SGLT2i or GLP1RA $\times$ stratification variable). Finally, we compared all
SGLT2i-treated patients with those treated with humanbased (liraglutide and dulaglutide) or exendin-based (exenatide and lixisenatide) GLP-1RAs, separately. The significance level was set to 0.05 for all analyses.

\section{RESULTS}

\section{Patient disposition and characteristics}

The study flowchart is shown in figure 1 . From a total population of 5242201 Italian citizens residing in the Veneto region and recorded as healthcare beneficiaries for at least 1 year, we identified 330193 (6.3\%) patients with a diagnosis of diabetes based on a validated claimsbased algorithm. ${ }^{21}$ Of these, 15530 patients initiated a new therapy with SGLT2i or GLP-1RA. We excluded patients who started GLP-1RA before 2014 (when SGLT2i received first marketing authorization approval in Italy), bringing the total to 12996 patients, of whom 7192 were new recipients of an SGLT2i and 5804 of a GLP-1RA. These two groups of patients showed small but significant differences in demographics and substantial differences in other clinical variables, including risk factors, comorbidities, and medications (online supplementary table S2). With PSM, we obtained two groups of 4298 patients each, which were very well balanced for all clinical characteristics collected at baseline and listed in table 1 .

SGLT2i were distributed as follows: $40 \%$ dapagliflozin, $50 \%$ empagliflozin, 10\% canagliflozin (ertugliflozin was not available in Italy). GLP-1RA were distributed as follows: $34 \%$ liraglutide, $14 \%$ exenatide, $48 \%$ dulaglutide, $4 \%$ lixisenatide (semaglutide and albiglutide were not available in Italy). On average, patients were 63 years old, $64 \%$ men, and had an estimated diabetes duration of 9.5 years. The majority of patients had hypertension $(82 \%)$ and dyslipidemia $(75 \%)$, but the prevalence of CVD was relatively low (18\%). Patients were previously being treated with an average of 2.3 classes of glucoselowering medications, $89 \%$ were on metformin, and $29 \%$ were also on insulin. Concomitant use of blood pressure and lipid lowering drugs was as frequent as in typical CVOTs.

Laboratory variables were available for a minority of patients in the database and, therefore, could not be incorporated into PSM. Nonetheless, we checked the balance between groups in the subset of matched patients for whom clinical-laboratory data were available $(n=1782$; $19.9 \%$ ). The two matched groups were very well balanced in terms of lipid profile and blood pressure, but there was a residual imbalance in fasting glucose and $\mathrm{HbAlc}$ (table 2), both of which were higher in the SGLT2i group.

\section{Cardiovascular outcomes}

The primary endpoint was 3P-MACE, that is, a composite of death, myocardial infarction, or stroke. We set a maximum follow-up length of 26 months, when only $20 \%$ of matched subjects remained in the study according to the 'AT' approach (ie, they were still following their initial therapy, were still visible in the claims database, 


\begin{tabular}{lccrr}
\hline Table 2 Clinical laboratory data after matching & & & & \\
\hline & SGLT2i (N=982) & GLP-1RA (N=800) & SMD* $^{*}$ & P value† \\
\hline Clinical-laboratory data & & & & \\
Fasting glucose (mg/dL) & $168.5(48.4)$ & $161.5(42.3)$ & 0.15 & 0.013 \\
\hline HbA1c (\%) & $8.0(1.1)$ & $7.8(0.9)$ & 0.21 & $<0.001$ \\
\hline Total cholesterol (mg/dL) & $168.6(37.8)$ & $168.8(37.2)$ & -0.01 & 0.497 \\
\hline HDL cholesterol (mg/dL) & $47.3(13.9)$ & $47.9(12.8)$ & -0.04 & 0.142 \\
\hline LDL cholesterol (mg/dL) & $93.6(33.6)$ & $93.0(32.4)$ & 0.02 & 0.326 \\
\hline Triglycerides $(\mathrm{mg} / \mathrm{dL})$ & $137.1(56.6)$ & $139.4(57.4)$ & -0.04 & 0.237 \\
\hline Systolic blood pressure $(\mathrm{mm} \mathrm{Hg})$ & $141.9(20.9)$ & $141.9(18.7)$ & $<0.01$ & 0.371 \\
\hline Diastolic blood pressure $(\mathrm{mm} \mathrm{Hg})$ & $81.2(10.6)$ & $81.1(10.7)$ & $<0.01$ & 0.450 \\
\hline
\end{tabular}

Clinical-laboratory data were collected from the visit closest to the index date. SMD values reported as ' $<0.01$ ' are, in fact, $<0.01$ and $>-0.01$. *SMDs (positive if SGLT2i greater).

†Mann-Whitney test.

GLP-1RA, glucagon-like peptide-1 receptor agonists; HbA1c, glycated hemoglobin; HDL, high density lipoprotein; LDL, low density lipoprotein; SGLT2i, sodium glucose cotransporter-2 inhibitors; SMD, standardized mean difference.

and had not yet experienced a cardiovascular outcome), and the median follow-up was 13 months (IQR 7-22). There were 257 3P-MACE (24.8 events/1000 personyears): 114 events occurred in the SGLT2i group and 143 in the GLP-1RA group, with corresponding incidences of 21.8 events/1000 person-years in the SGLT2i group and 27.9 in the GLP-1RA group. Cox proportional hazard regression yielded a statistically significant HR of 0.78 (95\% CI 0.61 to 0.99 ; $\mathrm{p}=0.043$ ) in favor of patients treated with SGLT2i over those treated with GLP-1RA. All secondary endpoints were observed less frequently in the SGLT2i group. The HRs for myocardial infarction $(0.72$; $95 \%$ CI 0.53 to $0.98 ; \mathrm{p}=0.035)$, HF $(0.59 ; 95 \%$ CI 0.35 to $0.99 ; \mathrm{p}=0.048)$, and hospitalization for CVD $(0.82 ; 95 \%$ CI 0.69 to $0.99 ; \mathrm{p}=0.037$ ) were significantly in favor of SGLT2i. These results, summarized in the top panel of figure 2, are also apparent from the Kaplan-Meier curves presented in online supplementary figure S1, where marked divergences are noticeable.

In the 'ITT' sensitivity analysis, the median follow-up was 18 months (IQR 10-26), but there was no control on whether subjects might have discontinued therapy with SGLT2i or GLP-1RA. The HRs for the primary outcome $(0.78,95 \%$ CI 0.62 to $0.97, p=0.027)$ and myocardial infarction $(0.75,95 \%$ CI 0.56 to $0.98, p=0.039)$ remained significantly in favor of SGLT2i. All other secondary outcomes exhibited the same trends in favor of SGLT2i seen in the 'AT' analysis but did not reach the significance level.

\section{Subgroup analyses}

We divided patients according to a history of preexisting CVD and ran two parallel sets of Cox regressions for the primary and secondary endpoints. HRs for most endpoints nominally favored the SGLT2i over the GLP-1RA group, with the exception of stroke and revascularization in the CVD-free subgroup, and death in the CVD-affected subgroup. The HRs for 3P-MACE
$(0.70 ; 95 \%$ CI 0.49 to 0.99$)$ and myocardial infarction $(0.63 ; 95 \%$ CI 0.42 to 0.94$)$ in the subgroup with CVD (figure 3), and hospitalization for HF in the one without (0.39; $95 \%$ CI 0.15 to 0.99$)$, significantly favored SGLT2i. The $p$ values associated with the interaction term in the interaction models were never below the nominal significance threshold of 0.05 .

In another subgroup analysis (online supplementary figure S2), we investigated HR for the primary endpoint according to baseline characteristics. All HRs for 3P-MACE at least nominally favored SGLT2i for all stratifications. Beginning a therapeutic regimen with SGLT2i, as compared with GLP-1RA, was associated with significantly lower risk of 3P-MACE in patients who were 65 or older, with pre-existing CVD, treated with statins, and treated with diuretics. However, after fitting Cox models with interactions, none of the HRs associated with the interaction terms was statistically significant. Both these subgroup analyses were likely underpowered and should mainly be viewed as descriptive.

In the comparison between all subjects treated with SGLT2i versus the two subgroups of patients treated with human-based and exendin-based GLP-1RA ( $\mathrm{n}=3506$ and $n=792$, respectively), all outcomes at least nominally favored SGLT2i, except stroke (HR 1.02) and revascularization (HR 1.04), which marginally and nonsignificantly favored patients treated with human-based GLP-1RA. Significantly lower rates of 3P-MACE, myocardial infarction, all-cause death, revascularizations, and hospitalization for cardiovascular causes were observed when SGLT2i were compared with exendin-based GLP1RA (online supplementary figure S3).

\section{Intermediate outcomes}

Patients with laboratory data available were subsets of the matched cohorts (982 and 800 in the SGLT2i and GLP1RA groups, respectively) who retained a good balance, with just a few variables showing statistically significant, 

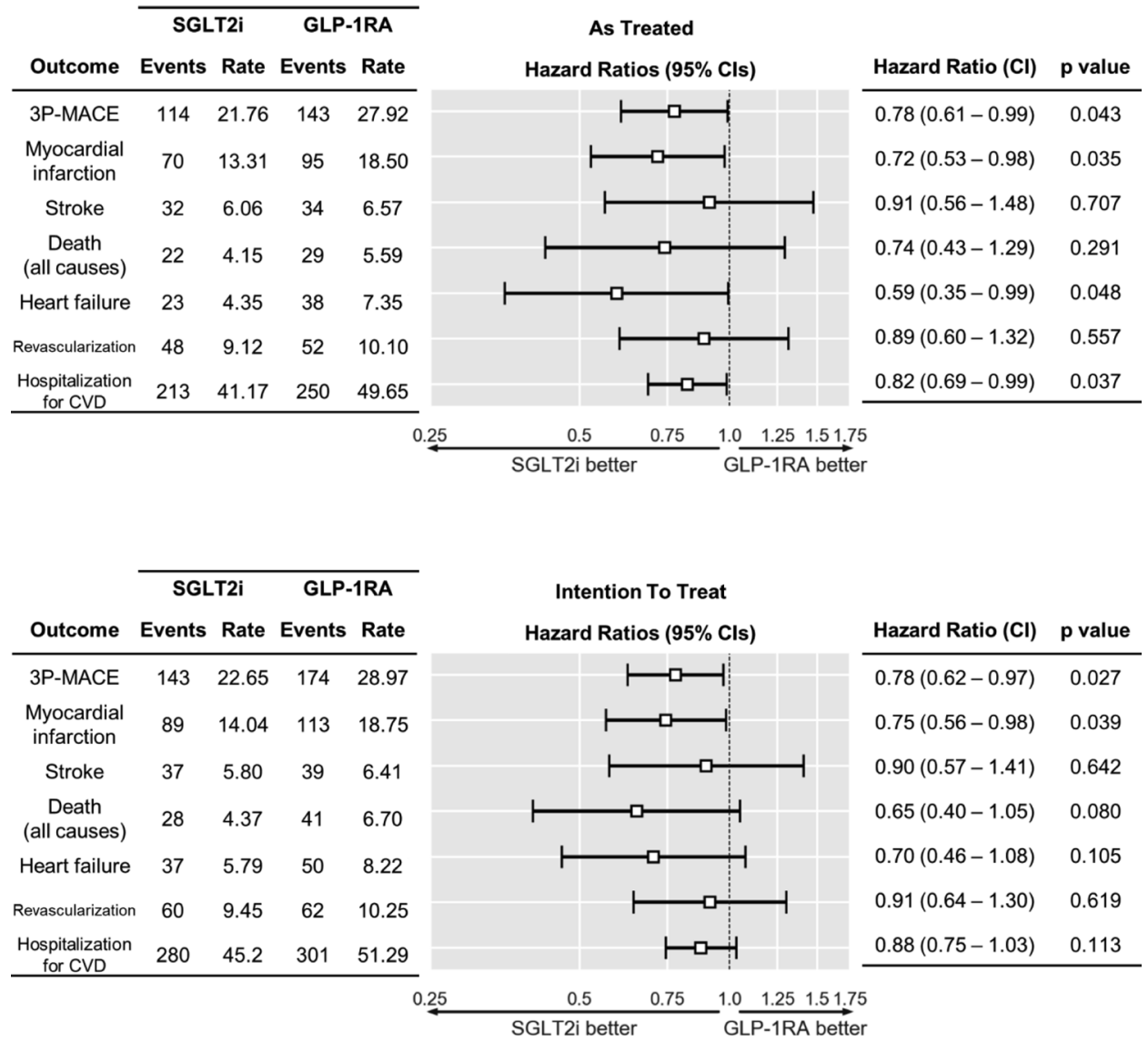

Figure 2 Outcome analysis. Results of Cox analysis on primary and secondary outcomes. Event rates are reported as number of events/1000 person-years. Top panel, AT analysis; bottom panel, ITT analysis. AT, as treated; CVD, cardiovascular disease; GLP-1RA, glucagon-like peptide-1 receptor agonists; ITT, intention to treat; SGLT2i, sodium glucose cotransporter-2 inhibitors; 3P-MACE, three-point major adverse cardiovascular events.

but mostly clinically irrelevant differences (online supplementary figure S4). We thus evaluated updated values of fasting glucose, HbAlc, lipid profile, and blood pressure and compared the change in these intermediate effectiveness parameters between the two groups. Patients in the GLP-1RA group improved HbAlc more than

\begin{tabular}{|c|c|c|c|c|c|c|c|}
\hline \multirow[b]{2}{*}{ Outcome } & \multicolumn{3}{|c|}{ N. of Subjects } & \multirow[b]{2}{*}{ Hazard Ratios (95\% Cls) } & \multirow[b]{2}{*}{ Hazard Ratio $(\mathrm{Cl})$} & \multirow[b]{2}{*}{ p value } & \multirow[b]{2}{*}{ p int. } \\
\hline & CVD & SGLT2i & GLP-1RA & & & & \\
\hline \multirow{2}{*}{ 3P-MACE } & yes & 786 & 759 & $\longmapsto$ & $0.70(0.49-0.99)$ & 0.049 & \multirow{2}{*}{0.420} \\
\hline & no & 3512 & 3539 & $\longmapsto$ & $0.86(0.61-1.21)$ & 0.382 & \\
\hline \multirow{2}{*}{$\begin{array}{l}\text { Myocardial } \\
\text { infarction }\end{array}$} & yes & 786 & 759 & $\longmapsto$ & $0.63(0.42-0.94)$ & 0.023 & \multirow{2}{*}{0.278} \\
\hline & no & 3512 & 3539 & 7 & $0.89(0.55-1.44)$ & 0.630 & \\
\hline \multirow{2}{*}{ Stroke } & yes & 786 & 759 & $\longrightarrow$ & $0.79(0.37-1.69)$ & 0.540 & \multirow{2}{*}{0.615} \\
\hline & no & 3512 & 3539 & & $1.01(0.54-1.90)$ & 0.967 & \\
\hline \multirow{2}{*}{$\begin{array}{c}\text { Death } \\
\text { (all causes) }\end{array}$} & yes & 786 & 759 & -1 & $1.00(0.42-2.40)$ & 0.998 & \multirow{2}{*}{0.407} \\
\hline & no & 3512 & 3539 & - & $0.61(0.30-1.26)$ & 0.184 & \\
\hline \multirow{2}{*}{ Heart failure } & yes & 786 & 759 & $\square$ & $0.74(0.40-1.39)$ & 0.352 & \multirow{2}{*}{0.273} \\
\hline & no & 3512 & 3539 & & $0.39(0.15-0.99)$ & 0.048 & \\
\hline \multirow{2}{*}{ Revascularization } & yes & 786 & 759 & - & $0.62(0.35-1.10)$ & 0.104 & \multirow{2}{*}{0.076} \\
\hline & no & 3512 & 3539 & & $1.27(0.73-2.21)$ & 0.401 & \\
\hline \multirow{2}{*}{$\begin{array}{l}\text { Hospitalization for } \\
\text { CVD }\end{array}$} & yes & 786 & 759 & $\mapsto$ & $0.82(0.61-1.10)$ & 0.183 & \multirow{2}{*}{0.942} \\
\hline & no & 3512 & 3539 & $\mapsto \square-1$ & $0.83(0.66-1.05)$ & 0.116 & \\
\hline & & & 0.1 & $\begin{array}{llll}0.5 & 0.751 .0 & 1.5 & 2.02 \\
\end{array}$ & & & \\
\hline
\end{tabular}

Figure 3 Outcome analysis by pre-existing CVD. Results of Cox analysis on primary and secondary outcomes, after stratification according to pre-existing CVD. In the figure, ' $p$ int.' refers to the interaction term (SGLT2i or GLP-1RA×CVD yes or no) in the adjusted model. CVD, cardiovascular disease; GLP-1RA, glucagon-like peptide-1 receptor agonists; N., number; SGLT2i, sodium glucose cotransporter-2 inhibitors; 3P-MACE, three-point major adverse cardiovascular events. 
SGLT2i $(-0.5 \%$ vs $-0.4 \%$; $\mathrm{p}=0.001$ when adjusting for baseline $\mathrm{HbA1c}$ ). The changes in systolic blood pressure, triglycerides, and HDL cholesterol were significantly in favor of SGLT2i (online supplementary table S3).

\section{Adverse events}

We collected data on a variety of typical adverse events known to be associated with either SGLT2i or GLP-1RA (online supplementary table S4). There were two cases of diabetic ketoacidosis, one on each group. Of the 16 amputations that occurred, 10 were registered in the SGLT2i group and 6 in the GLP-1RA group. No Fournier's gangrene was reported. Episodes of pancreatitis and pancreatic cancer were balanced between groups. Bone fractures occurred slightly and non-significantly more frequently in the SGLT2i than in the GLP-1RA group. Episodes of AKI occurred almost twice as frequently in the GLP-1RA than in the SGLT2i group, but the associated HR was non-significant. The number of GUTI, as retrieved from hospital discharge codes, was balanced between groups but was very small, most likely because such infections rarely required hospitalization; antibiotic prescriptions which were also not significantly different between groups (not shown).

\section{DISCUSSION}

This observational, retrospective, real-world study shows that T2D patients who initiated therapy with SGLT2i under routine care experienced lower rates of cardiovascular events than did similar patients who initiated GLP-1RA in the same period, geographic region, and healthcare setting.

The T2D population addressed in this study can be considered at relatively low risk, with only $18 \%$ of patients having CVD at baseline and with a 3P-MACE rate similar to that observed in the lowest-risk CVOTs with GLP-1RA (REWIND) and SGLT2i (DECLARE). ${ }^{45}$

All endpoint components, except stroke, contributed to the different cardiovascular outcome in the two groups. Superiority of SGLT2i over GLP-1RA was particularly evident for myocardial infarction and, as expected, for hospitalization for heart failure (HHF). Protection exerted by SGLT2i against myocardial infarction is consistent with a meta-analysis of placebo-controlled trials, ${ }^{2}$ and is supported by mechanistic studies in experimental models of atherosclerosis. ${ }^{24}{ }^{25}$ The observation that SGLT2i improved parameters directly related to cardiovascular risk (blood pressure and HDL) more than GLP-1RA supports this view. The lower rate of HHF in the SGLT2i group is consistent with CVOTs showing a marked effect of this class of drugs against $\mathrm{HHF}^{2}$, even in non-diabetic patients with prior $\mathrm{HF}^{26}$ which was stronger than with GLP-1RA. ${ }^{3}$ GLP-1RA therapy has been associated with lower risk of stroke, which was particularly strong for semaglutide and dulaglutide. ${ }^{527}$ Thus, it is not surprising that SGLT2i therapy was not associated with lower rates of stroke compared with GLP-1RA therapy.
All-cause mortality rates in our population were lower than in GLP-1RA or SGLT2i trials, ${ }^{45}$ which reflects the long life expectancy in Italy ${ }^{28}$ and limited statistical power for this endpoint.

Although subgroup analyses were underpowered, in the SGLT2i versus GLP-1RA group, rates of 3P-MACE and myocardial infarction seemed to be lower particularly among patients with prior CVD, whereas HHF rate was reduced more in patients without CVD. CVOTs also consistently show that the benefit of SGLT2i on 3P-MACE is mostly confined to patients with established CVD, whereas protection from HF occurs also in patients without CVD. ${ }^{2}$ Interestingly, except for HHF, better cardiovascular outcomes were consistently observed when SGLT2i were compared with exendin-based GLP1RA rather than with human-based GLP-1RA. This is consistent with non-inferiority of exendin-based GLP$1 \mathrm{RA}^{29} 30$ and superiority of human-based GLP-1RA ${ }^{5271}$ over placebo on the rate of 3P-MACE in the respective CVOTs.

The consensus on T2D management recommends SGLT2i and GLP-1RA as second-line agents, especially in patients with established cardiovascular or renal disease. ${ }^{32}$ The choice between these two classes of drugs is however based on subtle differences comparatively inferred from studies with different designs and populations. GLP-1RA would be preferred when atherosclerotic CVD predominates, whereas SGLT2i would be preferred when HF or renal disease predominate. Yet, HR for 3P-MACE was remarkably similar in trials with SGLT2i and GLP-1RA, ${ }^{23}$ suggesting no major difference in the overall cardiovascular protection these classes of drugs exert when tested against placebo. In addition, the populations investigated in most CVOTs are poorly representative of patients seen in routine clinical practice, thereby limiting generalizability. ${ }^{33}$ Thus, except when HF is already present ${ }^{26}$ no solid data support the choice between SGLT2i and GLP1RA for the management of T2D. For these reasons, head-to-head comparative assessment of cardiovascular effects of SGLT2i and GLP-1RA is clinically important. Our study results do not support the view that GLP-1RA predominantly counter atherosclerotic CVD, whereas SGLT2i mainly counter HF. Median observation time in our ITT analysis was the same as in the HARMONY (Albiglutide and cardiovascular outcomes in patients with type 2 diabetes and cardiovascular disease) trial, wherein albiglutide showed superiority versus placebo with respect to 3P-MACE and especially myocardial infarction. ${ }^{34} \mathrm{We}$ acknowledge, however, that in lower-risk populations such as the one addressed in our study, more time might be needed for an eventual anti-atherosclerotic effect of GLP-1RA to appear. Thus, future studies with longer follow-up will be of interest to address this issue.

This study's findings need to be interpreted in view of the limitations inherent to its retrospective non-randomized design. Due to confounding by indication, patients who initiated SGLT2i or GLP-1RA typically differed in many instances. Without addressing channeling bias, 
dissecting whether differential outcomes observed in the two cohorts are attributable to the drugs or to patient characteristics would be impossible. We ensured the best balance between the SGLT2i and GLP-1RA groups by means of PSM, a well-known strategy to obtain a pseudo-randomized condition, characterized by equal a posteriori probabilities of each subject being assigned to either treatment given baseline covariates. We derived all matching variables from administrative claims, while laboratory data provided a snapshot of baseline glucose, pressure, and lipid control in the population. Several other pieces of information were not available, such as socio-economic status, compliance, and life-style habits. An internal validation of the database comes from the observation that HbA1c declined more in patients who received GLP-1RA, whereas SGLT2i improved more blood pressure and HDL cholesterol, all of which are wellknown effects reported in trials. Importantly, focusing on patients newly treated with drugs that are equally positioned in the treatment algorithm, matching diabetes duration and the prior history of glucose lowering medications is the best strategy to minimize time lag bias. ${ }^{35}$

The study has other remarkable strengths. Follow-up duration was longer than in other studies of the same type ${ }^{836}$ thereby providing medium-term outcome information. SGLT2i and GLP-1RA could only be prescribed by diabetes specialist clinics in Italy during the study period, and we included only patients who initiated either class of drugs in the same period and in the same geographic area. Along with the balance achieved after PSM, this strategy created two very homogenous cohorts. Finally, since the overall benefit of a treatment strategy needs to weight safety and efficacy, we also collected information on adverse events, which is not commonly done in many retrospective studies. For instance, the trend for lower events of AKI in the SGLT2i group is in line with data from trials ${ }^{7}$ and real-world studies ${ }^{12}$ on kidney protection by SGLT2i and is reassuring against the risk of AKI that had prompted a boxed warning by the food and drug administration (FDA) in the label of SGLT2i. ${ }^{37}$

In summary, in the absence of evidence from randomized trials, our real-world study provides medium-level evidence that SGLT2i may be more effective than GLP1RA in preventing cardiovascular events in T2D patients seen during routine care, few of whom had pre-existing CVD, and who had been previously treated with complex regimens of glucose lowering medications.

Contributors Study design: EL, BDC, GS, LG, AA, GPF. Data collection and analysis: EL, GPF. Manuscript writing: EL, AA, GPF. Manuscript revision: BDC, GS, LG. All authors approved the final version to be published.

Funding This work was supported by Institutional grants from the University of Padova. Part of this work was supported by MIUR (Italian Ministry for Education) under the initiative 'Departments of Excellence' (Law 232/2016). EL is supported by Arsenàl.IT, Veneto's Research Centre for eHealth Innovation, through a PhD scholarship.

Competing interests AA received research grants, lecture or advisory board fees from Merck Sharp \& Dome, AstraZeneca, Novartis, Boeringher-Ingelheim, Sanofi, Mediolanum, Janssen, Novo Nordisk, Lilly, Servier, and Takeda. GPF received lecture fees or grant support from Abbott, AstraZeneca, Boehringer, Lilly, MerckSharp-Dome, Mundipharma, Novartis, Novo Nordisk, Sanofi, Servier.

Patient consent for publication Not required.

Ethics approval The study protocol conforms to the ethical guidelines of the 1975 Declaration of Helsinki. All the data used in this study were previously anonymized as per the Italian law concerning their usage for research and governance purposes. Study conduct was approved by the data owner board (Arsenàl.IT) and a formal ethics approval was deemed not necessary. Based on national regulations for retrospective studies on anonymized administrative claims, patients' informed consent was not collected.

Provenance and peer review Not commissioned; externally peer reviewed.

Data availability statement Data will be available from the corresponding author at a reasonable request.

Open access This is an open access article distributed in accordance with the Creative Commons Attribution Non Commercial (CC BY-NC 4.0) license, which permits others to distribute, remix, adapt, build upon this work non-commercially, and license their derivative works on different terms, provided the original work is properly cited, appropriate credit is given, any changes made indicated, and the use is non-commercial. See: http://creativecommons.org/licenses/by-nc/4.0/.

\section{ORCID iDs}

Barbara Di Camillo http://orcid.org/0000-0001-8415-4688 Giovanni Sparacino http://orcid.org/0000-0002-3248-1393 Gian Paolo Fadini http://orcid.org/0000-0002-6510-2097

\section{REFERENCES}

1 Gregg EW, Cheng YJ, Srinivasan M, et al. Trends in cause-specific mortality among adults with and without diagnosed diabetes in the USA: an epidemiological analysis of linked national survey and vital statistics data. Lancet 2018;391:2430-40.

2 Zelniker TA, Wiviott SD, Raz I, et al. Sglt2 inhibitors for primary and secondary prevention of cardiovascular and renal outcomes in type 2 diabetes: a systematic review and meta-analysis of cardiovascular outcome trials. Lancet 2019;393:31-9.

3 Kristensen SL, Rørth R, Jhund PS, et al. Cardiovascular, mortality, and kidney outcomes with GLP-1 receptor agonists in patients with type 2 diabetes: a systematic review and meta-analysis of cardiovascular outcome trials. Lancet Diabetes Endocrinol 2019;7:776-85

4 Wiviott SD, Raz I, Bonaca MP, et al. Dapagliflozin and cardiovascular outcomes in type 2 diabetes. N Engl J Med 2019;380:347-57.

5 Gerstein HC, Colhoun HM, Dagenais GR, et al. Dulaglutide and cardiovascular outcomes in type 2 diabetes (REWIND): a doubleblind, randomised placebo-controlled trial. Lancet 2019;394:121-30.

6 Bailey CJ. Choosing GLP-1 receptor agonists or SGLT-2 inhibitors by cardiorenal risk. Lancet Diabetes Endocrinol 2020;8:97-99.

7 Neuen BL, Young T, Heerspink HJL, et al. Sglt2 inhibitors for the prevention of kidney failure in patients with type 2 diabetes: a systematic review and meta-analysis. Lancet Diabetes Endocrinol 2019;7:845-54.

8 Patorno E, Pawar A, Franklin JM, et al. Empagliflozin and the risk of heart failure hospitalization in routine clinical care. Circulation 2019;139:2822-30.

9 Svanström H, Ueda P, Melbye M, et al. Use of liraglutide and risk of major cardiovascular events: a register-based cohort study in Denmark and Sweden. Lancet Diabetes Endocrinol 2019;7:106-14.

10 Udell JA, Yuan Z, Rush T, et al. Cardiovascular outcomes and risks after initiation of a sodium glucose cotransporter 2 inhibitor: results from the EASEL population-based cohort study (evidence for cardiovascular outcomes with sodium glucose cotransporter 2 inhibitors in the real world). Circulation 2018;137:1450-9.

11 Birkeland $\mathrm{KI}$, Jørgensen ME, Carstensen B, et al. Cardiovascular mortality and morbidity in patients with type 2 diabetes following initiation of sodium-glucose co-transporter-2 inhibitors versus other glucose-lowering drugs (CVD-REAL Nordic): a multinational observational analysis. Lancet Diabetes Endocrinol 2017;5:709-17.

12 Heerspink HJL, Karasik A, Thuresson M, et al. Kidney outcomes associated with use of SGLT2 inhibitors in real-world clinical practice (CVD-REAL 3): a multinational observational cohort study. Lancet Diabetes Endocrinol 2020;8:27-35.

13 Abdul-Ghani MA, Norton L, Defronzo RA. Role of sodium-glucose cotransporter 2 (SGLT 2) inhibitors in the treatment of type 2 diabetes. Endocr Rev 2011;32:515-31. 
14 Halimi S, Vergès B. Adverse effects and safety of SGLT-2 inhibitors. Diabetes Metab 2014;40:S28-34.

15 Monami M, Nreu B, Scatena A, et al. Safety issues with glucagonlike peptide-1 receptor agonists (pancreatitis, pancreatic cancer and cholelithiasis): data from randomized controlled trials. Diabetes Obes Metab 2017;19:1233-41.

16 Fadini GP, Sciannameo V, Franzetti I, et al. Similar effectiveness of dapagliflozin and GLP-1 receptor agonists concerning combined endpoints in routine clinical practice: a multicentre retrospective study. Diabetes Obes Metab 2019;21:1886-94.

17 Yang W, Zilov A, Soewondo P, et al. Observational studies: going beyond the boundaries of randomized controlled trials. Diabetes Res Clin Pract 2010;88 Suppl 1:S3-9.

18 Gerstein HC, McMurray J, Holman RR. Real-World studies no substitute for RCTs in establishing efficacy. Lancet 2019;393:210-1.

19 Regulation Concerning the Health Information Exchange [Regolamento in materia di fascicolo sanitario elettronico], 2019. Available: http://www.gazzettaufficiale.it/eli/id/2015/11/11/ $15 \mathrm{G} 00192 / \mathrm{sg}$

20 Portale Sanit Regione del Veneto - AUR - Anagrafe Unica Assistiti Regionale (Lotto B). Available: https://salute.regione.veneto.it/web/ bando-ssi/aur-anagrafe-unica-assistiti-regionale; Last [Accessed 7 Nov 2019].

21 Longato E, Di Camillo B, Sparacino G, et al. Diabetes diagnosis from administrative claims and estimation of the true prevalence of diabetes among 4.2 million individuals of the Veneto region (North East Italy). Nutr Metab Cardiovasc Dis 2020;30:84-91.

22 Deyo RA, Cherkin DC, Ciol MA. Adapting a clinical comorbidity index for use with ICD-9-CM administrative databases. J Clin Epidemiol 1992;45:613-9.

23 Charlson ME, Pompei P, Ales KL, et al. A new method of classifying prognostic comorbidity in longitudinal studies: development and validation. J Chronic Dis 1987;40:373-83.

24 Al-Sharea A, Murphy AJ, Huggins LA, et al. SGLT2 inhibition reduces atherosclerosis by enhancing lipoprotein clearance in $\mathrm{Ldlr}^{-/}$type 1 diabetic mice. Atherosclerosis 2018;271:166-76.

25 Han JH, Oh TJ, Lee G, et al. The beneficial effects of empagliflozin, an SGLT2 inhibitor, on atherosclerosis in $\mathrm{ApoE}^{-1-}$ mice fed a western diet. Diabetologia 2017;60:364-76.

26 McMurray JJV, Solomon SD, Inzucchi SE, et al. Dapagliflozin in patients with heart failure and reduced ejection fraction. $N$ Engl $J$ Med 2019;381:1995-2008.
27 Marso SP, Bain SC, Consoli A, et al. Semaglutide and cardiovascular outcomes in patients with type 2 diabetes. N Engl $\mathrm{J}$ Med 2016;375:1834-44.

28 GBD 2017 Italy Collaborators. Italy's health performance, 19902017: findings from the global burden of disease study 2017. Lancet Public Health 2019;4:e645-57.

29 Holman RR, Bethel MA, Mentz RJ, et al. Effects of once-weekly exenatide on cardiovascular outcomes in type 2 diabetes. $N$ Engl $J$ Med 2017;377:1228-39.

30 Pfeffer MA, Claggett B, Diaz R, et al. Lixisenatide in patients with type 2 diabetes and acute coronary syndrome. $N$ Engl $J$ Med 2015;373:2247-57.

31 Marso SP, Daniels GH, Brown-Frandsen K, et al. Liraglutide and cardiovascular outcomes in type 2 diabetes. N Engl J Med 2016;375:311-22.

32 Davies MJ, D'Alessio DA, Fradkin J, et al. Management of hyperglycemia in type 2 diabetes, 2018. A consensus report by the American diabetes association (ADA) and the European association for the study of diabetes (EASD). Diabetes Care 2018;41:2669-701.

33 Sciannameo V, Berchialla P, Orsi E, et al. Enrolment criteria for diabetes cardiovascular outcome trials do not inform on generalizability to clinical practice: the case of glucagon-like peptide-1 receptor agonists. Diabetes Obes Metab 2020;22:817-27.

34 Hernandez AF, Green JB, Janmohamed S, et al. Albiglutide and cardiovascular outcomes in patients with type 2 diabetes and cardiovascular disease (harmony outcomes): a double-blind, randomised placebo-controlled trial. Lancet 2018;392:1519-29.

35 Suissa S. Lower risk of death with SGLT2 inhibitors in observational studies: real or bias? Diabetes Care 2018;41:6-10.

36 Kosiborod M, Cavender MA, Fu AZ, et al. Lower risk of heart failure and death in patients initiated on sodium-glucose cotransporter-2 inhibitors versus other glucose-lowering drugs: the CVD-REAL study (comparative effectiveness of cardiovascular outcomes in new users of sodium-glucose cotransporter-2 inhibitors). Circulation 2017;136:249-59.

37 Food and Drug Administration. Fda drug safety communication: FDA strengthens kidney warnings for diabetes medicines canagliflozin (Invokana, Invokamet) and dapagliflozin (Farxiga, Xigduo XR), 2016. Available: https://www.fda.gov/drugs/drugsafety-and-availability/fda-drug-safety-communication-fdastrengthens-kidney-warnings-diabetes-medicines-canagliflozin [Accessed May 2020]. 